\title{
Quark mass dependence of baryon properties
}

\author{
Ulf-G. Meißner ${ }^{* i}$ \\ Helmholtz-Insitut für Strahlen- und Kernphysik \\ Universität Bonn \\ Nußallee 14-16 \\ D-53115 Bonn, Germany \\ and \\ Institut für Kernphysik (Theorie) \\ Forschungszentrum Jülich \\ D-52425 Jülich, Germany \\ E-mail: meissnerditkp.uni-bonn.de
}

I discuss the quark mass dependence of various baryon properties derived from chiral perturbation theory. Such representations can eventually be used as chiral extrapolation functions when lattice data at sufficiently small quark masses become available. The quark mass dependence is encoded in loop and contact term contributions, the latter given in terms of low-energy constants. I stress the importance of utilizing phenomenological input to constrain a certain class of low-energy constants and discuss the ensuing theoretical uncertainty for various baryon observables, like the nucleon and the baryon octet masses, the nucleon isovector anomalous magnetic moment and the axial-vector coupling of the nucleon. I stress the role of resonance decoupling and present first results for the delta mass based on an effective field theory in which the nucleon-delta mass splitting is counted as a small parameter. I also discuss briefly the pion mass dependence of the nuclear force as derived from chiral nuclear effective field theory.

XXIIIrd International Symposium on Lattice Field Theory

25-30 July 2005

Trinity College, Dublin, Ireland

\footnotetext{
* Speaker.

$\dagger$ This research is part of the EU Integrated Infrastructure Initiative Hadron Physics Project under contract number RII3-CT-2004-506078. Work supported in part by DFG (SFB/TR 16 "Subnuclear Structure of Matter").
} 


\section{Approaching the chiral limit in QCD}

Chiral perturbation theory (CHPT) is the appropriate tool to analyze the quark mass dependence of baryon properties. CHPT is the effective fi eld theory (EFT) of QCD, based on its symmetries and their realization [1, 2, 3. A central role in QCD is played by the spontaneously and explicitly broken chiral symmetry for the three light quark flavors up, down and strange. The vacuum is not invariant under this global $\mathrm{SU}(3)_{L} \times \mathrm{SU}(3)_{R}$ symmetry and the spectrum therefore contains eight almost massless pseudoscalar Goldstone modes. These are not exactly massless because the quark masses are non-zero but have very small values compared to typical strong interaction scales. The consequences of this explicit and spontaneous chiral symmetry breaking can be analyzed in the framework of CHPT. S-matrix elements and transition currents are expanded in small parameters - external momenta and quark (meson) masses with respect to the chiral symmetry breaking scale $\Lambda_{\chi} \simeq 1 \mathrm{GeV}$. Matter fi elds (that is all particles that are not Goldstone bosons) can also be considered in CHPT. A special role is played by the ground state baryons - they can not decay strongly and thus one is able to consistently deal with the new mass scale related to these fi elds - the chiral limit value of the baryon mass is neither non-vanishing nor small compared to the scale of chiral symmetry breaking $\Lambda_{\chi}$. Resonances, may they be meson or baryons, can not be incorporated so directly, one either has to resort to resummation schemes or consider additional small parameters that do not vanish for vanishing quark masses in the construction of the effective fi eld theory. For reviews on CHPT including also matter fi elds I refer to [4, $5,5, \square, \square]$ and the foundations of CHPT are discussed in depth in [曰]

We now consider the approach to the chiral limit. For the following discussion it is important to differentiate between the $\mathrm{SU}(2)$ and the $\mathrm{SU}(3)$ chiral limit (c.l.) of QCD. More precisely, in the $S U(2)$ c.l. we have $m_{u}, m_{d} \rightarrow 0$ with $m_{s}$ fi xed at its physical value and the heavy quarks decoupled; while in the $S U(3)$ c.l. $m_{u}, m_{d}, m_{s} \rightarrow 0$ with the heavy quarks decoupled. There are a few general observations about the chiral limit of QCD. First, S-matrix elements exist in the chiral limit for arbitrary momenta. Second, as it is well-established, the approach to the chiral limit is non-analytic in the quark masses $m_{q}$, leading to the famous chiral logarithms and other non-analytic behavior of certain observables. Third, in the fundamental paper [10] a decoupling theorem was derived. It states that the leading chiral non-analytic terms stem from pion (Goldstone boson) one-loop graphs coupled to pions (Goldstone bosons) or nucleons (ground state baryons). This theorem has various immediate consequences: resonances like the $\rho$, the $\Delta, \ldots$ decouple to leading order. This puts severe constraints on the construction of EFTs with resonance fi elds. Related to this is the observation that chiral limit of QCD and the large $N_{c}$ limit do not commute - we will come back to these issues later.

\section{One-loop representation of baryon observables}

We now consider a complete one-loop (fourth order) SU(2) calculation. Any nucleon observable $\mathscr{O}$ - given in terms of tree and loop diagrams - takes the form

$$
\mathscr{O}\left(M_{\pi}, F_{\pi} ; m_{0}, g_{A} ; c_{i}, d_{i}, e_{i}\right),
$$

where some of the quantities entering this expression are themselves quark mass dependent. Specifically, the pion mass and the pion decay constant are given by $M_{\pi}^{2}=\left(m_{u}+m_{d}\right) B_{0}+\mathscr{O}\left(m_{q}^{2}\right)$ and 
$F_{\pi}=F_{0}\left[1+\mathscr{O}\left(m_{q}\right)\right]$, where $B_{0}$ is related to the quark condensate and $F_{0}$ is the pion decay constant in the $\mathrm{SU}(2)$ chiral limit. Further, $m_{0}$ is the nucleon mass in the $\mathrm{SU}(2)$ c.l. and $g_{A}$ is the leading order (dimension one) axial-vector pion-nucleon coupling, $g_{A}=g_{0}\left[1+\mathscr{O}\left(m_{q}\right)\right]$. Also, the $c_{i}, d_{i}, e_{i}$ are low-energy constants (LECs) of the dimension two, three, and four chiral effective pion-nucleon Lagrangian $\mathscr{L}_{\text {eff }}$,

$$
\mathscr{L}_{\text {eff }}=\mathscr{L}_{\pi N}^{(1)}+\mathscr{L}_{\pi N}^{(2)}+\mathscr{L}_{\pi N}^{(3)}+\mathscr{L}_{\pi N}^{(4)}+\ldots
$$

where the superscript $(n)$ denotes the chiral dimension (the purely pionic part of the Lagrangian is not made explicit here). The complete effective Lagrangian is given in [11]. For later use, I display the dimension one and two terms:

$$
\begin{aligned}
\mathscr{L}_{\pi N}^{(1)}= & \bar{\Psi}\left(i \not D-m_{0}+\frac{1}{2} g_{0} u \gamma_{5}\right) \Psi \\
\mathscr{L}_{\pi N}^{(2)}= & \sum_{i=1}^{7} \bar{\Psi} c_{i} O_{i}^{(2)} \Psi=\bar{\Psi}\left(c_{1}\left\langle\chi_{+}\right\rangle+c_{2}\left(-\frac{1}{8 m_{0}^{2}}\left\langle u_{\mu} u_{v}\right\rangle D^{\mu v}+\text { h.c. }\right)+c_{3} \frac{1}{2}\langle u \cdot u\rangle\right. \\
& \left.\quad+c_{4} \frac{i}{4}\left[u_{\mu}, u_{v}\right] \sigma^{\mu v}+c_{5} \tilde{\chi}_{+}+c_{6} \frac{1}{8 m_{0}} F_{\mu \nu}^{+} \sigma^{\mu v}+c_{7} \frac{1}{8 m_{0}}\left\langle F_{\mu v}^{+}\right\rangle \sigma^{\mu v}\right) \Psi \\
\mathscr{L}_{\pi N}^{(3)}= & \sum_{i=1}^{23} \bar{\Psi} d_{i} O_{i}^{(3)} \Psi, \quad \mathscr{L}_{\pi N}^{(3)}=\sum_{i=1}^{118} \bar{\Psi} e_{i} O_{i}^{(4)} \Psi
\end{aligned}
$$

with $U(x)=u^{2}(x)$ collects the pion fi elds $\pi(x)=\vec{\tau}^{a} \cdot \vec{\pi}^{a}(x), \Psi(x)$ the isodoublet nucleon fi eld, $u_{\mu}=i u^{\dagger} D_{\mu} U u^{\dagger}=-i \partial_{\mu} \pi / F_{0}+\ldots$ and $D_{\mu}$ the chiral covariant derivative. The $O_{i}^{(n)}$ are monomials of chiral dimension $n$. Furthermore, $\chi_{+}$parameterizes the external scalar source including the quark masses, $\chi_{+} \sim 2 B_{0} \operatorname{diag}\left(m_{u}, m_{d}\right)+\ldots, F_{\mu v}^{+}$is the chiral and gauge covariant photon fi eld strength tensor, and \langle\rangle denotes the trace in flavor space (for more details, see [1, 11]). The LECs can be grouped into two distinct classes. Class I are the so-called dynamical LECs $\sim \partial_{\mu}^{2}, \partial_{\mu}^{4}, \ldots$ which are non-vanishing in the chiral limit. Many of these are accessible via phenomenology, that is through the analysis of scattering data or decay processes. Class II LECs, the so-called symmetry breakers $\sim m_{q}, m_{q}^{2}, m_{q} \partial_{\mu}^{2}, \ldots$, vanish in the c.l. and they can best be obtained by varying the quark masses, i.e. in lattice simulations (see also the discussion in [12]). Note that the mixed LECs parameterizing operators with quark mass insertions and derivatives are counted as symmetry breakers because at fi xed pion mass these can be absorbed in the values of certain dynamical LECs (with the exception of the leading mixed LECs at dimension three). The dimension two effective Lagrangian in Eq. (2.3) has two symmetry breakers and fi ve dynamical LECs, from the latter two can only be determined in the presence of external fi elds. At dimension three, we have 4 symmetry breakers and 19 dynamical LECs - in fact, the symmetry breakers are necessarily of the mixed type and thus are easier available from phenomenological analysis as most other LECs of that type. Finally, at dimension four, we have 25 symmetry breakers (most of them of mixed type) and 93 dynamical LECs (most of them containing external fi elds). For more details, see [1]]. As we will see in the following, while the total number of LECs increases rapidly, this is not the case for most observables - this will become evident when I discuss the nucleon mass, the isovector magnetic moment or the axial-vector coupling constant. 


\section{Analysis of baryon properties}

Chiral perturbation theory supplies the quark mass expansion of any given observable to the required accuracy - provided the expansion parameter is suffi ciently small. There is an important interplay between chiral loops and the LECs parameterizing the contact interactions. Mostly, one employs dimensional regularization to perform the necessary renormalization of the loop contributions in a symmetry-preserving manner (although other schemes are viable, too). While an observable $\mathscr{O}$ does not depend on the scale of dimensional regularization $\lambda$, that is $d \mathscr{O} / d \lambda=0$, this is in general not the case for the individual contributions from the loops or the counter terms. An example of this is the isovector charge radius of the nucleon as discussed in [13]. It is therefore absolutely necessary to include all terms in harmony with the underlying symmetries, and not just the chiral logs or other non-analytic terms. Also, it has become evident over the years that in baryon CHPT one has to perform complete one-loop (fourth order) calculations to obtain a precise enough representation at physical pion/kaon masses (in SU(3), this statement is not true in general). For reviews, see [14, 15].

Before continuing, let me make the following disclaimer: I will discuss continuum extrapolations, but no fi nite lattice spacing or fi nite volume effects Also, I will not discuss (partial) quenching, that means all lattice data are taken as full QCD. Further, I will be mostly interested in the range of applicability (theoretical uncertainty) rather than precision fi ts - for reasons that become apparent in the following - and fi nally, I eschew models here - end of disclaimer.

\subsection{Quark mass expansion of the nucleon mass in $\mathrm{SU}(2)$ and $\mathrm{SU}(3)$}

Consider the expansion of the nucleon mass in the light quark mass $\hat{m}=m_{u}=m_{d}$ with $m_{s}$ fi xed. By means of two-flavor CHPT, this is mapped onto an expansion in the pion mass. Note that we consider strong isospin violation $\left(m_{u} \neq m_{d}\right)$ later. The fourth order calculation gives [16]

$$
m_{N}=m_{0}-4 c_{1} M_{\pi}^{2}-\frac{3 g_{A}^{2} M_{\pi}^{3}}{32 \pi F_{\pi}^{2}}+k_{1} M_{\pi}^{4} \ln \frac{M_{\pi}}{m_{N}}+k_{2} M_{\pi}^{4}+\mathscr{O}\left(M_{\pi}^{5}\right),
$$

with $m_{0}$ the nucleon mass in the chiral SU(2) limit and $k_{1}$ and $k_{2}$ are combinations of second $\left(c_{1}, c_{2}, c_{3}\right)$ and fourth order LECs $\left(e_{1}\right), k_{1}=-\left(3 / 32 \pi^{2} F_{\pi}^{2}\right)\left(-8 c_{1}+c_{2}+4 c_{3}+g_{A}^{2} / m_{N}\right)$ and $k_{2}=$ $-4 e_{1}+\left(3 / 128 \pi^{2} F_{\pi}^{2}\right)\left(c_{2}-2 g_{A}^{2} / m_{N}\right)$, respectively. The scale of dimensional regularization was set equal to the nucleon mass, $\lambda=m_{N}$. For simplicity, I have neglected in Eq.(3.1) the difference between the physical value of the pion mass and its leading term in the quark mass expansion, $M_{\pi}^{2}=M^{2}\left[1+\mathscr{O}\left(m_{q}\right)\right]$ (for details, see e.g. [17]). Note also that the dimension two LECs are scale-independent in any mass-independent regularization scheme, while the fourth order LEC is scale-dependent, $e_{1}=e_{1}(\lambda)$. It is important to realize that the LECs $c_{1}, c_{2}, c_{3}$, and $e_{1}$ are strongly constrained from pion-nucleon scattering and to some extent form peripheral nucleon-nucleon scattering. More specifi cally, the chiral expansion of the $\pi N$ amplitude converges best inside the Mandelstam triangle, however, to determine the values of the LECs, one must compare to a dispersive analysis of the scattering data. This was done in [18]. Alternatively, one can use the chiral representation of the amplitude in the physical region close to threshold, here one is faced with certain inconsistencies in the data basis. The most detailed fi ts based on these data were performed in [19]. One can also pick out certain threshold parameters that are particularly sensitive to certain 
LECs, as it was done in [20]. In addition, the peripheral partial waves in elastic nucleon-nucleon are sensitive to the two-pion exchange and thus to some of the $c_{i}$. In this case, one has a large data basis but must subtract the dominant one-pion exchange, see [21, 22] (see also Ref. [23] - which, however, does not enter my average). Putting all this information together, we obtain for the $c_{i}$ $(i=1, \ldots, 4)$ (all values in $\left.\mathrm{GeV}^{-1}\right)$

$$
c_{1}=-0.9_{-0.2}^{+0.5}, \quad c_{2}=3.3 \pm 0.2, \quad c_{3}=-4.7_{-1.0}^{+1.2}, \quad c_{4}=3.5_{-0.2}^{+0.5}, \quad-2 c_{1}+\frac{c_{2}}{4}+c_{3}=-2.1_{-1.1}^{+1.3} \text {. }
$$

A few remarks are in order. First, the symmetry breaker $c_{1}$ and the isoscalar combination (last number) are least accurately determined - as expected. Also, the LEC $e_{1}$ is only badly determined from $\pi N \rightarrow \pi N$ in the threshold region, the analysis of [24] gives a negative value of natural size. It is also important to notice that the numerical values of the LECs $c_{i}$ can be well understood in terms of resonance saturation (baryon and meson resonance excitations), in particular, the fairly large values of $c_{2,3}\left(c_{4}\right)$ are generated mostly by $\Delta(1232)(\rho)$ exchange [20]. Before discussing the quark mass dependence of the nucleon mass as given by Eq. (3.1), it is important to point out that for the physical pion mass, the higher order corrections are small. More precisely, for the central values of the LECs given in Eq. (3.2) (and using $e_{1}=-1 \mathrm{GeV}^{-3}$ ), the contribution quadratic, cubic and quartic in $M_{\pi}$ amounts to $70,-17$ and $-4 \mathrm{MeV}$, respectively. It was even shown in [25] that the fi fth order corrections are tiny (at the physical point). I will return to this issue of convergence later.
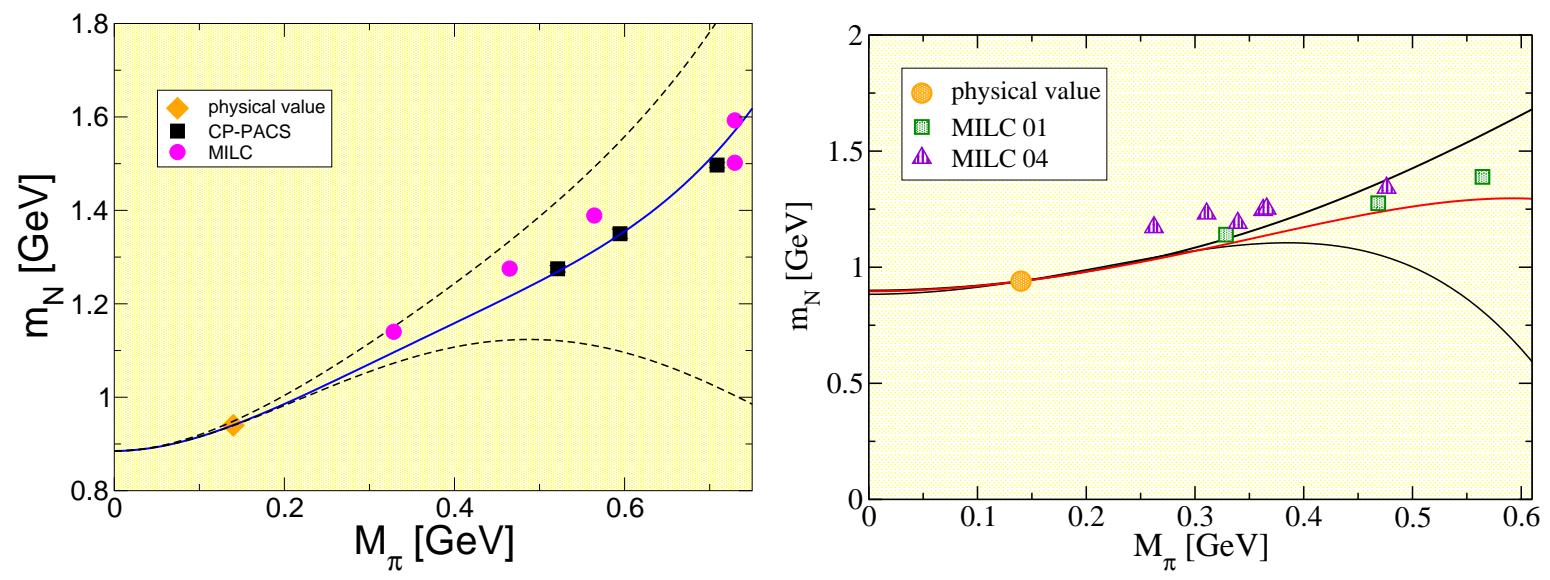

Figure 1: Pion mass dependence of the nucleon mass. Left panel: SU(2) analysis. The blue solid line refers to the best fit and the dashed black lines to the theoretical uncertainty as discussed in the text. Red circles: MILC data [26], black squares: CP-PACS data [27]. Right panel: SU(3) analysis. The red line gives the best fit as discussed in the text and the black lines give the theoretical uncertainty under the constraint that the nucleon mass takes its physical value for the physical quark masses. Green squares: MILC 2001 data [26], purple triangles: MILC 2004 data [28]

In the left panel of Fig. 1] we show the results from Ref. [13]. A best fit to the MILC 2001 26] and the CP-PACS data [27] is obtained with $c_{1}=-0.9, c_{2}=3.2, c_{3}=-3.5\left(\right.$ all in $\left.\mathrm{GeV}^{-1}\right)$ and $e_{1}\left(m_{N}\right)=-1 \mathrm{GeV}^{-3}$. Note that this is not a chiral extrapolation in the true sense of the word because the constraint that the physical value of $m_{N}$ is obtained at the physical pion mass was imposed. Note also that the MILC data are from an SU(3) simulation, however, they have also 
given an $\mathrm{SU}(2)$ reference value for one pion mass that differs only little from the corresponding $\mathrm{SU}(3)$ result. The resulting $\mathrm{SU}(2)$ c.l. value of the nucleon mass is $m_{0} \simeq 0.88 \mathrm{GeV}$. The dashed lines in the fi gure reflect the theoretical uncertainty from the variation of the LECs, consequently, we obtain a moderate/large theoretical uncertainty for $M_{\pi}$ above $400 / 550 \mathrm{MeV}$. Clearly, at higher pion masses the higher order terms become too important for such fi ts to make sense. For related work on the nucleon mass in SU(2), see [29, 30]. The theoretical uncertainty in the determination of the pion mass dependence of the nucleon mass is also discussed in [31].

Next, I consider the extension to the three-flavor case. Before discussing the nucleon mass, some general remarks are in order. As compared to the $\mathrm{SU}(2)$ analysis, one has more fi elds and operator structures in the EFT, and consequently, more LECs. As examples, consider the leading order axial meson-baryon coupling in $\mathrm{SU}(2)$ and $\mathrm{SU}(3)$

$$
\frac{1}{2} g_{A} \bar{\psi} u_{\mu} \gamma^{\mu} \gamma^{5} \psi \rightarrow D\left\langle\bar{B} \gamma^{\mu} \gamma^{5}\left\{u_{\mu}, B\right\}\right\rangle+F\left\langle\bar{B} \gamma^{\mu} \gamma^{5}\left[u_{\mu}, B\right]\right\rangle
$$

or the leading symmetry breakers

$$
c_{1} \bar{\psi}\left\langle\chi_{+}\right\rangle \psi \rightarrow b_{0}\langle\bar{B} B\rangle+b_{D}\left\langle\bar{B}\left\{\chi_{+}, B\right\}\right\rangle+b_{F}\left\langle\bar{B}\left[\chi_{+}, B\right]\right\rangle,
$$

with $\Psi$ the nucleon doublet and $B$ the baryon octet coupled to the pion triplet and the Goldstone boson octet, respectively. Note that the LEC $b_{0}$ can be absorbed in the value of the octet baryon mass in the chiral limit, but need to be kept separately when one also analyses the pion- and kaonnucleon sigma terms [32]. These various operators in SU(2) and SU(3) are related by matching conditions - in the $\mathrm{SU}(2)$ case the strange quark effects are buried in certain local operators. Such matching relations have been worked out in [33], to leading order, one fi nds, e.g.

$$
\begin{array}{ll}
\text { nucleon mass in the } \mathrm{SU}(3) \text { chiral limit : } & \tilde{m}_{0}=m_{0}\left[1+\mathscr{O}\left(m_{s}\right)\right], \\
\text { leading axial coupling : } & g_{A}=D+F+\mathscr{O}\left(m_{s}\right), \\
\text { leading symmetry breaker : } & c_{1}=b_{0}+\frac{1}{2}\left(b_{D}+b_{F}\right)+\mathscr{O}\left(\sqrt{m_{s}}\right) .
\end{array}
$$

The full matching conditions to fourth order in the chiral expansion are given in [33]. These are important constraints that should be implemented in any SU(3) analysis. I return to the nucleon mass. Ref. [33] contains the complete fourth order expressions (including isospin breaking terms) for the baryon octet masses and sigma terms. Based on that work, in [34] fi ts to the MILC 2001 data based on two different regularization schemes (cut-off and dimensional regularization) were performed. Again, the constraint to obtain the physical value of the nucleon mass at the physical quark masses was imposed. The dimension two LECs called $b_{i}$ were taken from the earlier work in [35] and the three combinations of the dimension four LECs (called $d_{i}$ ) were varied to obtain a best description of the three-flavor MILC 2001 data, see the red line in the right panel of Fig. 1. In the fi gure are also shown the MILC 2004 data [28] at lower quark masses - these can not be fi tted with the constraint (as already noted by the MILC collaboration using a simplifi ed mass formula). Taking into account also the kaon mass dependence of the nucleon mass and the uncertainty due to the MILC 2004 data, we obtain the following ranges for various (isoscalar) quantities:

$$
\begin{array}{lr}
\mathrm{SU}(3) \text { c.l. value of } \mathrm{m}_{\mathrm{N}}: & 710 \mathrm{MeV} \lesssim \tilde{m}_{0} \lesssim 1070 \mathrm{MeV}, \\
\text { Pion - nucleon sigma term : } & 39.5 \mathrm{MeV} \lesssim \sigma_{\pi N}(0) \lesssim 46.7 \mathrm{MeV}, \\
\text { Strangeness fraction : } & 0.07 \lesssim y \lesssim 0.22,
\end{array}
$$



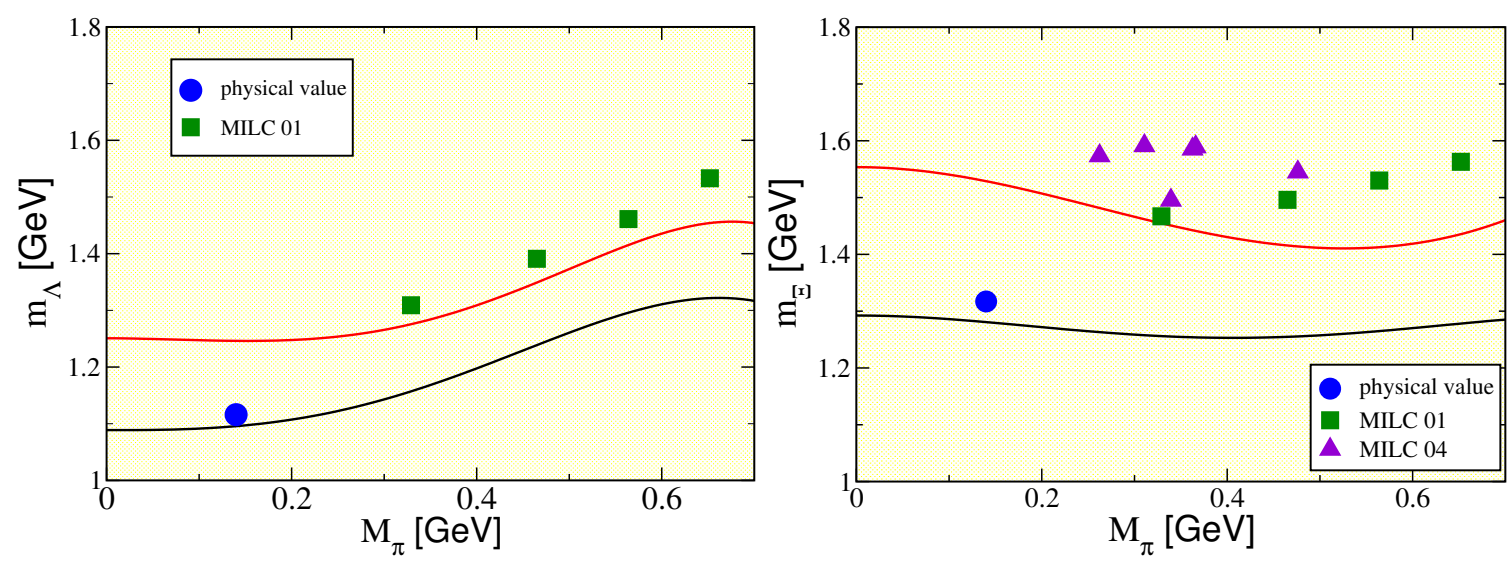

Figure 2: Pion mass dependence of the $\Lambda$ mass (left panel) and of the $\Xi$ mass (right panel). Green boxes/purple triangles: MILC 2001/2004 data. The blue circle denotes the physical value of the corresponding baryon mass. For further details, see the text.

with $\sigma_{\pi N}(0)=\left\langle N(p)\left|\hat{m}\left({ }^{-} u u+\bar{d} d\right)\right| N(p)\right\rangle$ (with $N(p)$ a nucleon state of momentum $p$ ) and the strangeness fraction in the proton, $y=2\left\langle\left. p\right|^{-} s s \mid p\right\rangle /\left\langle\left. p\right|^{-} u u \bar{d} d \mid p\right\rangle$, can be obtained from $\sigma_{\pi N}(0)=$ $\sigma_{0} /(1-y)$ and using $\sigma_{0}=(37 \pm 6) \mathrm{MeV}$ from [35]. It was concluded in [34] that for pion masses less than $400 \mathrm{MeV}$ the convergence of the chiral expansion is fi ne and that it is acceptable for masses below $550 \mathrm{MeV}$. This agrees with the findings of the SU(2) analysis in [13].

\subsection{Quark mass expansion of the baryon octet masses}

We now consider the other octet members with strangeness $(\Lambda, \Sigma, \Xi)$ based on the work of Ref. [34]. In that paper, the fourth order LECs were fi tted to the nucleon mass only, that means the GellMann-Okubo relation for the baryon masses was not imposed. Consequently, some of the masses come out somewhat off their empirical values. More precisely, while the $\Sigma$ mass is well reproduced, the $\Lambda$ and $\Xi$ masses come out by about $10-15 \%$ too high. To get a handle on the theoretical accuracy, we also use the values for the $d_{i}$ from [35], in that case all masses are exactly reproduced. The resulting prediction for the pion mass dependence of the $\Lambda$ and the $\Xi$ in comparison to the MILC data are shown in Fig. 2. The red/black lines refer to the optimal set of the LECs from the nucleon mass fit t/to the LECs from [3\$]. We note in particular that the pion mass dependence for the $\Xi$ is much flatter as one would expect from the MILC data. This is not unexpected - the $\Xi$ only contains one valence light quark and should thus be less sensitive to variations in the pion mass. Clearly, one could improve this description by fi tting directly to these particles. Still, the pion mass dependence of the $\Xi$ as given by the MILC data is a mystery to be resolved.

So far, I have considered the isospin limit $m_{u}=m_{d}$. I briefly discuss the implications of unequal light quark masses for the strong neutron-proton mass difference. In the $\mathrm{SU}(2)$ case, it is given to leading order by

$$
\left(m_{n}-m_{p}\right)^{\text {strong }}=4 c_{5} B_{0}\left(m_{u}-m_{d}\right)+\mathscr{O}\left(\left(m_{u}-m_{d}\right)^{2}\right)
$$

with $c_{5}$ the dimension two LEC accompanying the operator $\tilde{\chi}_{+}=\chi_{+}-\frac{1}{2}\left\langle\chi_{+}\right\rangle \sim B_{0}\left(m_{u}-m_{d}\right)$, see also Eq. (2.3). Utilizing the Cottingham sum rule to estimate the electromagnetic splitting, one 
fi nds [20]

$$
c_{5}=-0.09 \pm 0.01 \mathrm{GeV}^{-1} .
$$

The small value of this LEC is a reflection of the suppression of isospin violation in QCD, which scales as $\left(m_{u}-m_{d}\right) / \Lambda_{\chi}$. (Note that Weinberg obtained a slightly larger value in his seminal paper because he employed SU(3) relations [36]). The fourth order corrections to Eq. (3.7) have been worked out, see e.g. [37]. In SU(3), the expression for the strong neutron-proton mass is much more complicated, since one has to treat $\pi^{0}-\eta$ and $\Lambda-\Sigma^{0}$ mixing in terms of the parameter $\tan 2 \varepsilon=(\sqrt{3} / 2)\left(m_{u}-m_{d}\right) /\left(m_{s}-\hat{m}\right)$. Again, one can derive a matching condition [33], to leading order it reads (for related work, see the pioneering analysis in [38] and also [39])

$$
c_{5}=b_{D}+b_{F}+\mathscr{O}\left(m_{s}, m_{s} \ln m_{s}\right) .
$$

Needless to say that for obtaining precise mass splittings, one needs to consider also the electromagnetic corrections (for an early analysis in the meson sector, see [40]).

\subsection{Quark mass expansion of other nucleon properties}

Besides the nucleon mass and sigma term, chiral extrapolations for various other nucleon observables based on chiral perturbation theory (or extensions thereof) have been considered in the literature, e.g. nucleon magnetic moments and the electromagnetic radii [41, 42, 43] or the isovector axial-coupling constant [44, 45, 46]. I will not review these in detail but rather make some more general remarks on the present status of extrapolation functions derived from baryon chiral perturbation theory. There are two important issues which require special attention:

1 Given the scarcity of data at low pion masses (say below $M_{\pi} \simeq 400 \mathrm{MeV}$ ), one should perform global fi ts to a variety of observables at suffi ciently small quark masses. The important point is that the LECs - the parameters of these fi ts - relate many observables, they are the same for all processes and can therefore not be determined independently. Furthermore, one should incorporate as much phenomenological input as possible, in particular for the dynamical LECs, see the discussion of the nucleon mass in the preceeding sections. Needless to say that one must be in a regime where higher order terms stay suffi ciently small. Attempts to go beyond this regime are necessarily model-dependent, see e.g. [47] and references therein.

2 Results should be independent of the regularization scheme, since these can differ only by higher order terms if symmetries are properly implemented. For example, the so-called infrared regularization (IR) of baryon CHPT [17] resums all kinetic energy insertions $\vec{p}^{2} / 2 m_{N}$ whereas the latter are expanded order-by-order in the heavy baryon approach (there are a few circumstances for which the heavy baryon approach does not converge, but these are not of relevance here). Similarly, cut-off schemes or fi nite-range-regulators as employed by the Adelaide group, see e.g. [48], resum certain higher order corrections (if implemented properly). For small pion masses as defi ned above, all these schemes must give the same result.

Let me illustrate these issues on the example of the nucleon isovector anomalous magnetic moment $\kappa_{v}$. It has been worked out to fourth order in [49] (the pertinent Feynman diagrams are shown in the left panel of Fig. 3)

$$
\kappa_{v}=c_{6}-16 m_{N} M_{\pi}^{2} e_{106}+\kappa_{v}^{\mathrm{loop}, 3}+\kappa_{v}^{\mathrm{loop}, 4},
$$




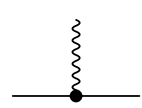

(a)

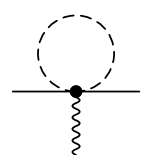

(d)

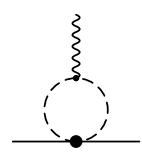

(g)

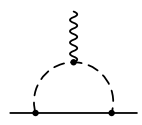

(b)

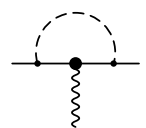

(e)

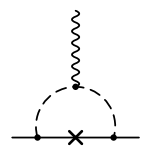

(h)

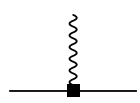

(c)

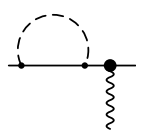

(f)

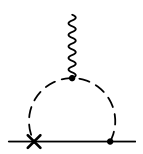

(i)

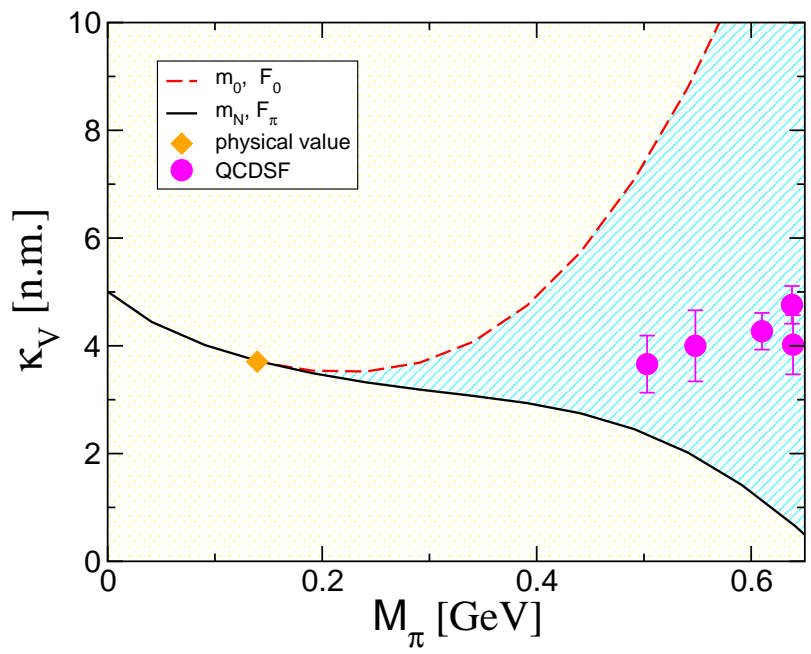

Figure 3: Left panel: Second (a), third (b) and fourth (c-i) order diagrams contributing to $\kappa_{v}$. The solid/dashed/wiggly lines stand for nucleons/pions/photons, respectively. The $\bullet(\mathbf{\square})$ denotes a dimension two (four) insertion. The $\times$ denotes an dimension two insertion with fixed coefficients. Right panel: Pion mass dependence of $\kappa_{v}$ as described in the text: The upper (lower) line corresponds to the c.l. (physical) values of the pion decay constant and the nucleon mass. The diamond represents the physical value of $\kappa_{v}$. The data are from QCDSF [43].

where I have made explicit the contributions from the dimension two $\sim c_{6}$ (diagram (a) in Fig. 3) and four $\sim e_{106}$ contact interactions (diagram (c) in Fig. 3). Here, $c_{6}$ is nothing but the anomalous magnetic moment in the chiral limit, $\kappa_{v}^{0}=c_{6}$. The fourth order loop contribution depends explicitely on the dimension two LECs $c_{2}$ (the tadpole graph (g) in Fig. B), $c_{4}$ (the tadpole graph (d) in Fig. 3), and $c_{6}$ (diagrams (e) and (f) in Fig. B). Two of these are constrained from pionnucleon scattering, cf. Eq. (3.2). The expression for $\kappa_{v}$ contains the nucleon mass $m_{N}$ and the pion decay constant $F_{\pi}$ (in the loop contributions). These quantities depend themselves on the pion mass, $m_{N}=m_{N}\left(M_{\pi}\right)$ and $F_{\pi}=F_{\pi}\left(M_{\pi}\right)$. To the order we are working, one can always replace the parameters in the effective Lagrangian ( $m_{0}$ and $F_{0}$, the pertinent c.l. values, see e.g. Eq. (2.3) by their physical values, the differences being of higher order in the chiral expansion. Thus, replacing $\left\{m_{0}, F_{0}\right\}$ by $\left\{m_{N}, F_{\pi}\right\}$ in the formula Eq. (3.10) is a way of investigating the convergence of the series, and I will do this here for $\kappa_{v}{ }^{1}{ }^{1}$ The explicit expression for the pion mass expansion of $m_{N}$ is given in Eq. (3.1) and $F_{\pi}$ to $\mathscr{O}\left(M_{\pi}^{4}\right)$ can be taken from [50] (see also Ref. [51] and references therein)

$\frac{F_{\pi}}{F_{0}}=1+X\left[\tilde{L}+\tilde{\ell}_{4}\right]+X^{2}\left[-\frac{3 \tilde{L}^{2}}{4}+\left(-\frac{7 \tilde{L}_{1}}{6} \tilde{\ell}_{1}-\frac{4 \tilde{\ell}_{2}}{3}+\tilde{\ell}_{4}-\frac{29}{12}\right)+\frac{\tilde{\ell}_{3} \tilde{\ell}_{4}}{2}-\frac{\tilde{\ell}_{1}}{12}-\frac{\tilde{\ell}_{2}}{3}-\frac{13}{192}+\tilde{r}_{F}(\lambda)\right]$

where $\tilde{L}=\log \left(\lambda^{2} / M_{\pi}^{2}\right), X=M_{\pi}^{2} /\left(16 \pi^{2} F_{0}^{2}\right)$ and $\tilde{r}_{F}(\mu)$ is a combination of dimension six LECs. I use $\lambda=0.5 \mathrm{GeV}$ and $\tilde{r}_{F}(\lambda=0.5 \mathrm{GeV})=3$ in the following. The NLO LECs $\tilde{\ell}_{i}$ are tabulated in [50]. Guided by the trend of the QCDSF data [43] and imposing the constraint that $\kappa_{v}=3.71$ [n.m.] at the physical pion mass, we set $c_{6}=5$ and $e_{106}=0.45$. The resulting theoretical uncertainty due to the two choices for the pion decay constant and the nucleon mass are shown in Fig. 3. As before,

\footnotetext{
${ }^{1}$ I am grateful to Véronique Bernard for providing me with these results.
} 
below pion masses of about $400 \mathrm{MeV}$, the theoretical uncertainty is modest, but above it quickly increases - so that a model-independent extraction of $\kappa_{v}$ using the particular data shown in the fi gure is not possible.

Let me now consider the axial-vector coupling $g_{A}$, which was much discussed in the recent literature, see e.g. [44, 45, 46]. I present here some fi rst results of an on-going study with Véronique Bernard [52]. There are two main issues concerning this particular observable. First, to my opinion, the existing data are at too high pion masses to draw defi nite conclusions about the range of applicability of the chiral extrapolation functions or the role of explicit spin-3/2 degrees of freedom. Second, there is a much more direct problem with the pion mass dependence of $g_{A}$. It has been worked out to fourth order fi rst in [53]. Using the standard form of the effective pion-nucleon Lagrangian (see Eq.(2.3) and Ref. [11]) it reads

$$
\begin{aligned}
g_{A}=g_{0}\left[1+\frac{4 M_{\pi}^{2}}{g_{0}}(\right. & \left.d_{16}(\lambda)-\frac{\frac{1}{2} g_{0}+g_{0}^{3}}{16 \pi^{2} F_{0}^{2}} \ln \frac{M_{\pi}}{\lambda}\right)-\frac{g_{0}^{2} M_{\pi}^{2}}{16 \pi^{2} F_{0}^{2}} \\
& \left.+\frac{M_{\pi}^{3}}{24 \pi F_{0}^{2} m_{0}}\left(3+3 g_{0}^{2}-4 m_{0} c_{3}+8 m_{0} c_{4}\right)\right]+\mathscr{O}\left(M_{\pi}^{4}\right) .
\end{aligned}
$$

The dimension three coupling constant $d_{16}$ can be determined from the process $\pi N \rightarrow \pi \pi N$ [54], however, not very accurately. For a typical value of the LEC $\bar{d}_{16}=-1.76 \mathrm{GeV}^{-2}$, the correction quadratic in the pion mass is $\Delta g_{A}^{(2)} \simeq 0.16$, which is of typical size for an $\mathrm{SU}(2)$ correction (note that $\bar{d}_{16}$ is the renormalized value of $d_{16}$ at $\lambda=M_{\pi}$, see [19]). It was, however, already pointed out in [53] that the corrections $\sim M_{\pi}^{3}$ are already quite sizeable for the physical pion mass. Using the central values of the LECs $c_{3}$ and $c_{4}$, one fi nds $\Delta g_{A}^{(3)} \simeq 0.32$, using $F_{0}=86.5 \mathrm{MeV}$ [50], $m_{0}=$ $880 \mathrm{MeV}$ [ [13] and $g_{0}=1.2$. This is a fairly large correction for an $\mathrm{SU}(2)$ quantity. It can be traced back mostly to the particularly large combination of the dimension two LECs $-4 m_{0} c_{3}+8 m_{0} c_{4} \simeq$ 41 , and thus one can not expect this representation to be very accurate. This is further corroborated in Fig. $\sharp$, where the pion mass dependence of $g_{A}$ is shown for various values of the LEC $\bar{d}_{16}$ and two values of $g_{0}=1.0$ and $g_{0}=1.3$, respectively. The strong increase of $g_{A}\left(M_{\pi}\right)$ with growing $M_{\pi}$ is due to the large correction cubic in the pion mass - this clearly narrows the window for applying Eq. (3.12) as a chiral extrapolation functions to even smaller pion masses than it is the case for the nucleon mass or the isovector magnetic moment discussed earlier. It should also be stressed that using an extrapolation function based only on the third order calculation (i.e. including the terms up to quadratic order in $M_{\pi}$ ) is insuffi cient - one does not even capture the largest correction at the physical value of the pion mass. On the other hand, it is conceivable that even higher order corrections are again of natural size - this, however, needs to be checked by an explicit calculation. A more detailed discussion of these issues will be given in [52] (see also [44]).

\subsection{Quark mass expansion of the $\Delta$ mass}

The $\Delta(1232)$ is the most important baryon resonance. It is almost degenerate in mass with the nucleon and couples strongly to pions, nucleons and photons. It was therefore argued early that spin-3/2 (decuplet) states should be included in baryon chiral perturbation theory [55]. In that paper and subsequent works use was made of the heavy baryon approach, which treats the baryons as static sources. However, special care has to be taken about the decoupling of resonances in the 


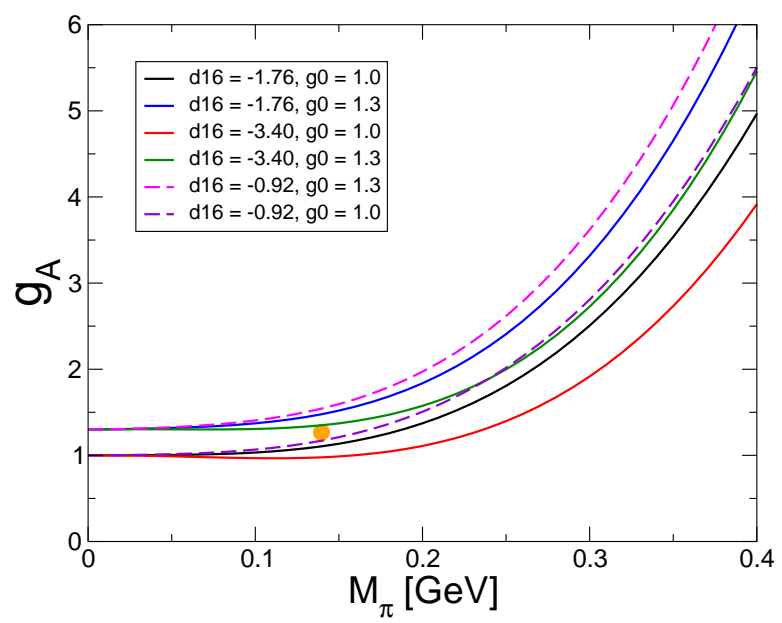

Figure 4: Pion mass dependence of the axial-vector coupling for various values of the LEC $\bar{d}_{16}=$ $-0.92,-1.72,-3.4 \mathrm{GeV}^{-2}$ and the chiral limit value of $g_{A}, g_{0}=1.0$ and 1.3 , respectively. The filled orange circle denotes the physical value of $g_{A}$ at the physical pion mass.

chiral limit, as discussed in Sec. 1 - this is frequently ignored in literature. This approach can be systematized by counting the nucleon-delta mass splitting as an additional small parameter, so one expands in the generic small parameter $\varepsilon$ [[5], with

$$
\varepsilon \in\left\{\frac{p}{\Lambda_{\chi}}, \frac{M_{\pi}}{\Lambda_{\chi}}, \frac{m_{\Delta}-m_{N}}{\Lambda_{\chi}}\right\} .
$$

The corresponding power counting is called the "small scale expansion" (SSE). It is important to note that the mass splitting $m_{\Delta}-m_{N}$ does not vanish in the chiral limit, therefore the explicit inclusion of the delta is a phenomenological extension of CHPT. More recently, it was realized that for certain considerations/processes a Lorentz-invariant formulation of baryon chiral perturbation theory is advantageous, particular related to the spin sector probed e.g. in doubly virtual Compton scattering. A particularly elegant scheme to perform covariant calculations is the so-called "infrared regularization" (IR) of [17]. In [57] a consistent extension of the infrared regularization method in the presence of spin-3/2 was given. It was in particular shown that the contribution of the non-propagating spin-1/2 components of the Rarita-Schwinger fi eld can be completely absorbed in the polynomial terms stemming from the most general effective chiral Lagrangian (see also the recent work in [58]).

Here, I report on some results obtained in [59]. In that paper, the nucleon and the delta mass as well as the corresponding sigma terms were analyzed to fourth order in the small parameter $\varepsilon$ (for related early work on this problem see [60]). These explicit representations of $m_{N}$ and $m_{\Delta}$ serve as chiral extrapolation functions to analyze the lattice data from the MILC collaboration [26] for unphysical pion masses as low as $M_{\pi} \simeq 350 \mathrm{MeV}$ (note that we treat the SU(3) data as if they were SU(2) - the same comments as in Sec. 3.1 apply). Clearly, such extrapolation functions based on chiral perturbation theory cease to make sense at too large values of the quark (pion) masses, 
but as we will demonstrate later, we can nicely capture the trend of these data. Before proceeding, it should be stressed that in the theory with explicit spin-3/2 degrees of freedom one has more oeprators and thus more LECs than in nucleon CHPT - similar to the case when going from SU(2) to SU(3) in CHPT discussed earlier.

The corresponding expansions to $\mathscr{O}\left(\varepsilon^{4}\right)$ for the nucleon and the delta mass take the form [59]

$$
\begin{gathered}
m=m_{0}-4 c_{1} M_{\pi}^{2}-4 M_{\pi}^{2} \Delta_{0} D_{1}-D_{2} \Delta_{0}^{3}-4 e_{1}(\lambda) M_{\pi}^{4}-E_{1} \Delta_{0}^{4}-4 M_{\pi}^{2} \Delta_{0}^{2} E_{2}+m^{\mathrm{N}-\text { loop }}+m^{\Delta-\text { loop }}, \\
m_{\Delta}=m_{\Delta}^{0}-4 a_{1} M_{\pi}^{2}-4 M_{\pi}^{2} \Delta_{0} D_{1}^{\Delta}-D_{2}^{\Delta} \Delta_{0}^{3}-4 e_{1}^{\Delta}(\lambda) M_{\pi}^{4}-E_{1}^{\Delta} \Delta_{0}^{4}-4 M_{\pi}^{2} \Delta_{0}^{2} E_{2}^{\Delta}+m_{\Delta}^{\mathrm{N}-\text { loop }}+m_{\Delta}^{\Delta-\text { loop }},
\end{gathered}
$$

where I have made explicit the contribution from the various contact interactions. Consider fi rst the nucleon. Its representation looks very similar to the one given in Eq. (3.1). There are, however, some important differences. First, the appearance of the delta in the loops also generates additional renormalizations $\sim \Delta_{0}^{n}(n \geq 1)$ (accompanied by combinations of dimension three $\left(D_{1,2}, D_{1,2}^{\Delta}\right.$ and dimension four $\left(E_{1,2}, E_{1,2}^{\Delta}\right)$ LECs) that can be absorbed in the LECs of the theory without delta (this is discussed in detail in [61]). These LECs can be chosen in such away that the delta contribution to the nucleon mass starts at $\mathscr{O}\left(M_{\pi}^{4}\right)$. Second, since there is an explicit delta-loop contribution, the values of the LECs $c_{i}$ differ from the ones determined earlier by the explicit delta contribution that has been worked out in [20]. Third, the delta loops introduce new parameters, in particular the leading axial nucleon-delta coupling called $c_{A}$ and the dimension two couplings $b_{1}$ and $b_{6}$ from the effective $\pi N \Delta$ Lagrangian appear in the expression for $m_{N}$, utilizing

$$
\begin{aligned}
& \mathscr{L}_{\pi N \Delta}^{(1)}=c_{A}\left\{\bar{\psi}_{i}^{\mu} w_{\mu}^{i} \psi_{N}+\text { h.c. }\right\} \\
& \mathscr{L}_{\pi N \Delta}^{(2)}=\bar{\psi}_{i}^{\mu}\left\{b_{3} i w_{\mu \nu}^{i} \gamma^{v}+\frac{b_{6}}{m_{0}} i w_{\mu \nu}^{i} i D^{v}+\ldots\right\} \psi_{N}+\text { h.c. },
\end{aligned}
$$

with $w_{\mu}^{i}=\left\langle\tau^{i} u_{\mu}\right\rangle / 2$ and $w_{\mu \nu}^{i}=\left\langle\tau^{i}\left[D_{\mu}, u_{v}\right]\right\rangle / 2$, where $\tau^{i}, i=1,2,3$ denote the Pauli matrices in isospin space. Here, $\psi_{i}^{\mu}$ denotes the spin-3/2 fi eld (for more details, see [2Q]). The corresponding fourth-order diagrams for the delta self-energy are shown in the left panel of Fig. 5. In particular, one has dimension two and four LECs corresponding to the ones in the nucleon mass case, for completeness I display the pertinent terms of the dimension two effective Lagrangian,

$$
\mathscr{L}_{\pi \Delta}^{(2)}=-\bar{\psi}_{i}^{\mu}\left\{\left[a_{1}\left\langle\chi_{+}\right\rangle-\frac{a_{2}}{4 m_{0}^{2}}\left\{\left\langle u_{\alpha} u_{\beta}\right\rangle D^{\alpha} D^{\beta}+\text { h.c. }\right\}+\frac{a_{3}}{2}\left\langle u^{2}\right\rangle+\ldots\right] g_{\mu \nu} \delta^{i j}+\ldots\right\} \psi_{j}^{\nu},
$$

so that the $a_{i}$ of the delta correspond to the $c_{i}$ of the nucleon and similarly $e_{1}^{\Delta}$ is a combination of dimension four LECs as it is the case for $e_{1}$ discussed earlier.

We are now in the position to analyze the pion mass dependence of the nucleon and delta mass given in Eqs. (3.14 3.14). They contain a certain number of LECs, some of which are (not very accurately) known from the study of pion-nucleon scattering in the heavy baryon SSE [22]. In addition to the known values at the physical point we take the data from MILC [26] for the nucleon and the delta as function of the pion mass and try to describe these with LECs of natural size. Such a description is indeed possible, as shown in Fig. 5. So we do not intend least-square fi ts here but rather try to fi nd out whether the existing data shown in this fi gure can be consistently described by 

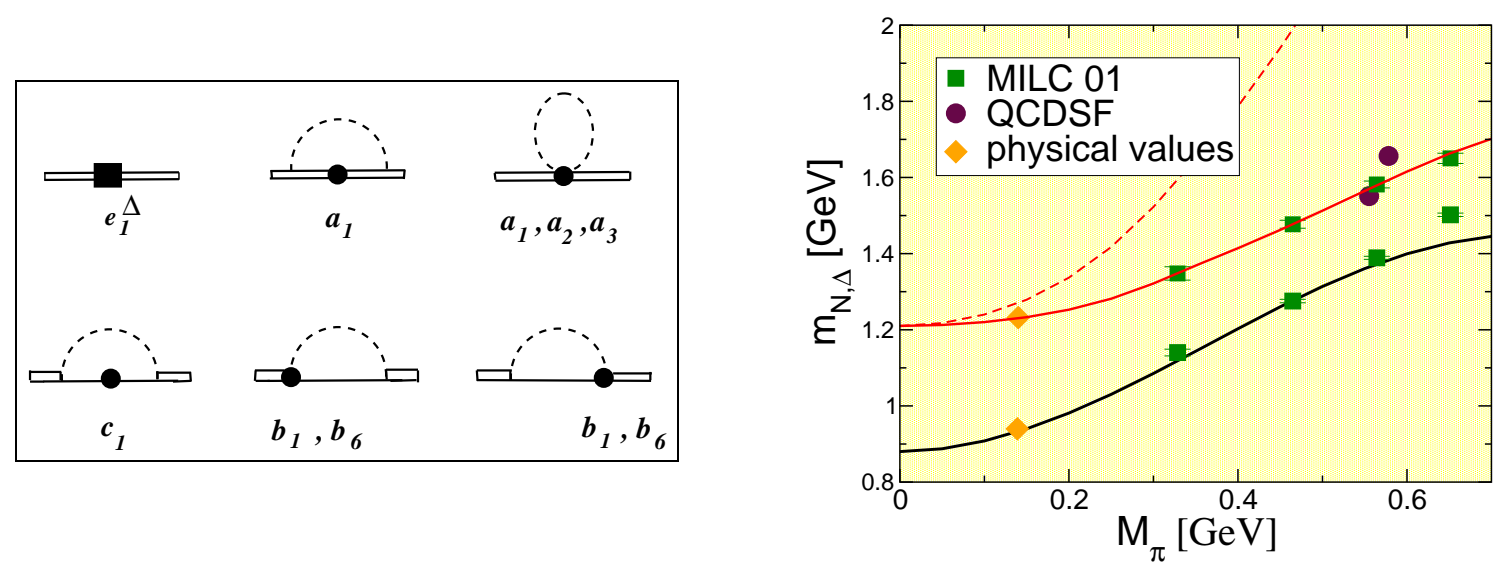

Figure 5: Left panel: Fourth order contribution to the delta mass. Shown are the contact interactions, pion loops (dashed lines) with intermediate nucleons (single lines) and intermediate deltas (double lines). The relevant LECs are also shown. Right panel: The nucleon mass (black line) and the (real part of the) delta mass (red line) as a function of the pion mass. The filled diamonds denote their physical values at the physical pion mass. The dashed line is the chiral extrapolation for the $\Delta$ based on $\mathrm{SU}(6)$ as explained in the text. The filled squares and circles are the MILC data [26]. The filled triangles are the recent data from QCDSF [63].

our mass formulas with LECs of natural size. We stress again that a more refi ned analysis of e.g. pion-nucleon scattering in the covariant SSE is mandatory to put stringent constraints on certain combinations of the LECs. The parameters corresponding to these curves are: (i) the $N \Delta$ mass splitting in the chiral limit, $\Delta_{0}=0.33 \mathrm{GeV}$, indicating a slightly larger $N \Delta$ mass splitting in the chiral limit than at the physical point. (ii) The LECs from the pion-nucleon Lagrangian are given by $c_{1}=-0.8 \mathrm{GeV}^{-1}$ (which is within the uncertainty of the values determined in e.g. [18]) and $e_{1}=c_{2}=0, c_{3}=0.5 \mathrm{GeV}^{-1}$. The small values of $c_{2,3}$ are consistent with resonance saturation studies of [20] and the fi ts in [67]. The fourth-order LEC $e_{1}$ induces the largest uncertainty - even a small value of $e_{1}$ leads to a sizeable contribution at larger pion masses. (iii) The three axial $N \Delta$ LECs are $c_{A}=1.1, b_{3}=0.75 \mathrm{GeV}^{-1}$ and $b_{6}=-0.75$. (iv) The axial $\Delta \Delta$ coupling is found to be $h_{A}=2$, which is not far from the $\mathrm{SU}(6)$ or large- $N_{C}$ value $h_{A}=9 g_{A} / 5=2.28$. Furthermore we get $e_{1}^{\Delta}=-1 \mathrm{GeV}^{-3}, a_{1}=-0.3 \mathrm{GeV}^{-1}, A / 4+B=a_{2} / 4+a_{3}+\ldots=0.5 \mathrm{GeV}^{-1}$. These are all natural values. It is interesting to note that $a_{1}$ is markedly smaller than $c_{1}$, although both couplings should be equal in the $\mathrm{SU}(6)$ limit. Still, it is interesting to study the strict $\mathrm{SU}(6)$ limit. In that case, one would have $a_{1}=c_{1}=-0.8 \mathrm{GeV}^{-1}$ and $h_{A}=2.28$. As can be seen from the dashed line in Fig. 5 , the assumption of strict SU(6) symmetry is clearly at odds with the MILC data, indicating that $a_{1}$ and $c_{1}$ indeed seem to have different values. Also shown in Fig. 5 are the recent QCDSF data for $m_{\Delta}$, which were not used in the fi t but are nicely consistent with our extrapolation function. Note also that the QCDSF data are based on two-flavor simulations and are not very different from the MILC data in the region of overlap. This further supports our assumption on the treatment of the MILC data. We stress again that the resulting values of the LECs are to be considered indicative and a more detailed analysis employing also constraints from other physical processes should follow. From the small value of the LEC $a_{1}$ one immediately deduces that the $\pi \Delta$ sigma term appears to be signifi cantly smaller than its nucleon cousin because at leading order in the quark mass expansion we have $\sigma_{\pi N}=-4 c_{1} M_{\pi}^{2}+\ldots$ and $\sigma_{\pi \Delta}=-4 a_{1} M_{\pi}^{2}+\ldots$. It is clear that this interesting observation 
deserves further study. Finally we note that the sigma term for the nucleon resulting from this "rough" fi $t$ is found as

$$
\sigma_{\pi N}=48.9 \mathrm{MeV}
$$

to order $\varepsilon^{4}$. We note that this value is consistent with the classical result of Ref. [54], which was confi rmed in [1\$] in a heavy baryon CHPT analysis of pion-nucleon scattering and in [29] in a CHPT analysis of lattice data. It is also in agreement with the recent CHPT analysis of the threeflavor MILC data, see 3.1. For the $\pi \Delta$ sigma term we get $\sigma_{\pi \Delta}=20.6 \mathrm{MeV}$. Note that model studies give slightly larger values between 28 and $35 \mathrm{Mev}$ [65, 66]. Again, these results need to be refi ned and bolstered by more detailed precise fi ts to the lattice data including also error and correlation analysis including also lattice data on other observables - the mass data alone are not suffi cient to precisely pin down all parameters. Such an analysis, however, is not yet available. Note also that chiral extrapolation functions for the baryon octet and decuplet masses in partially quenched QCD based on an EFT with deltas are discussed in [67, 68].

\section{Quark mass dependence of nuclear forces}

Because of the smallness of the up and down quark masses, one does not expect signifi cant changes in systems of pions or pions and one nucleon when the quark masses are set to zero (with the exception of well understood chiral singularities like e.g. in the pion radius or the nucleon polarizabilities). The situation is more complicated for systems of two (or more) nucleons. Here, I report on some work [69] that is mostly concerned with the properties of the deuteron and the $\mathrm{S}$-wave scattering lengths as a function of the quark (pion) mass. These questions are not only of academic interest, but also of practical use for interpolating results from lattice gauge theory. E.g. the S-wave scattering lengths have been calculated on the lattice using the quenched approximation [70]. Another interesting application is related to imposing bounds on the time-dependence of some fundamental coupling constants from the two-nucleon (NN) sector, as discussed in [71].

To address these issues, consider the chiral two-nucleon potential at next-to-leading order (NLO). It is given in terms of one- and two-pion exchanges (OPE and TPE, respectively) and four-nucleon contact interactions

$$
V=V_{\mathrm{OPE}}+V_{\mathrm{TPE}}+V_{\text {contact }} .
$$

In this approach, we have to deal with explicit and implicit quark mass dependences. This can be most easily understood by looking at the OPE and contact interactions:

$$
\begin{aligned}
V_{\mathrm{OPE}} & =\left(-\frac{g_{A}^{2}}{4 F_{\pi}^{2}}+\ldots \bar{d}_{16}+\ldots\right) \vec{\tau}_{1} \cdot \vec{\tau}_{2} \frac{\left(\vec{\sigma}_{1} \cdot \vec{q}\right)\left(\vec{\sigma}_{2} \cdot \vec{q}\right)}{\vec{q}^{2}+M_{\pi}^{2}} \\
V_{\text {contact }} & =C_{S}+M_{\pi}^{2} D_{S}+\left(C_{T}+M_{\pi}^{2} D_{T}\right)\left(\vec{\sigma}_{1} \cdot \vec{\sigma}_{2}\right)+\ldots
\end{aligned}
$$

The OPE exhibits both types of quark mass dependences. The pion propagator becomes Coulomblike in the chiral limit, $1 /\left(\vec{q}^{2}+M_{\pi}^{2}\right) \rightarrow 1 / \vec{q}^{2}$. The implicit pion mass dependence enters at NLO through the pion-nucleon coupling constant (note that the quark mass dependence of the nucleon mass only enters at NNLO) expressed through the pion mass dependence of $g_{A} / F_{\pi}$ in terms of the quantity

$$
\Delta=\left(\frac{g_{A}^{2}}{16 \pi^{2} F_{\pi}^{2}}-\frac{4}{g_{A}} \bar{d}_{16}+\frac{1}{16 \pi^{2} F_{\pi}^{2}} \bar{l}_{4}\right)\left(M_{\pi}^{2}-\tilde{M}_{\pi}^{2}\right)-\frac{g_{A}^{2} \tilde{M}_{\pi}^{2}}{4 \pi^{2} F_{\pi}^{2}} \ln \frac{\tilde{M}_{\pi}}{M_{\pi}}
$$




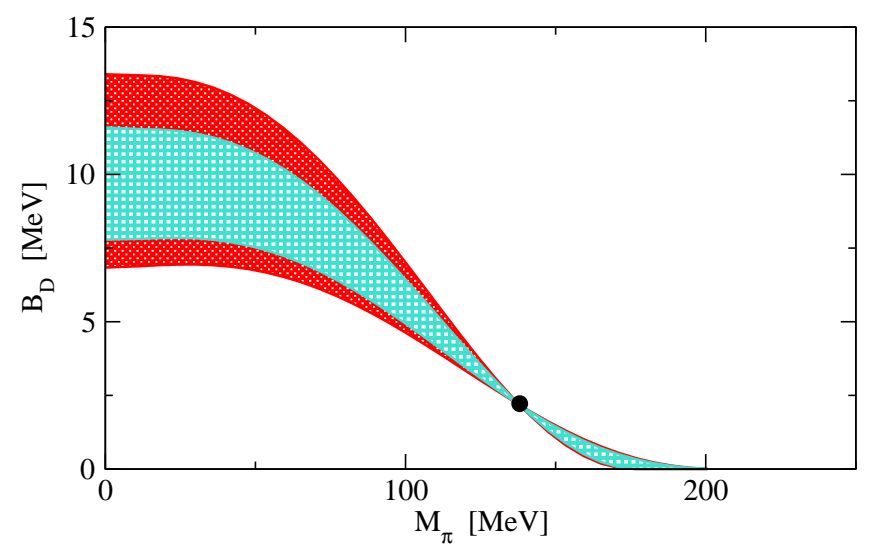

Figure 6: Deuteron BE versus the pion mass. The shaded areas show the allowed values. The light shaded band corresponds to our main result with the uncertainty due to the unknown LECs $D_{S, T}$. The dark shaded band gives the additional uncertainty due to the uncertainty of $\bar{d}_{16}$. The heavy dot shows the BE for the physical case $\tilde{M}_{\pi}=M_{\pi}$.

Here $\bar{l}_{4}$ and $\bar{d}_{16}$ are LECs related to pion and pion-nucleon interactions, and the value of the varying pion mass is denoted by $\tilde{M}_{\pi}$ in order to distinguish it from the physical one denoted by $M_{\pi}$. In particular, $\bar{d}_{16}$ has been determined in various fi ts to describe $\pi N \rightarrow \pi \pi N$ data, see [5]]. The four-nucleon contact terms exhibit an explicit quark mass dependence, These NLO $M_{\pi}^{2}$ corrections to the leading operators $\sim C_{S}, C_{T}$ are parameterized by the LECs $D_{S}$ and $D_{T}$, respectively. These LECs can at present only be estimated using dimensional analysis and resonance saturation [72]. For the explicit expressions of the TPE, see [69].

It is now straightforward to solve the Lippmann-Schwinger equation for the bound and scattering states utilizing the properly regularized chiral EFT potential, Eq. (4.2). The deuteron binding energy (BE) as a function of the pion mass is shown in Fig.6. We fi nd that the deuteron is stronger bound in the chiral limit than in the real world,

$$
B_{\mathrm{D}}^{\mathrm{c} .1 .}=9.6 \pm 1.9_{-1.0}^{+1.8} \mathrm{MeV}
$$

where the fi rst indicated error refers to the uncertainty in the value of $\bar{D}_{{ }^{S_{1}}}$ and $\bar{d}_{16}$ being set to its average value while the second indicated error shows the additional uncertainty due to the uncertainty in the determination of $\bar{d}_{16}$. Note that in [73] a larger variation of the LECs $D_{S, T}$ was considered, leading to the possibility that the deuteron can become unbound in the chiral limit. Also, for massless pions, the higher partial waves are no longer suppressed by the centrifugal barrier, $\delta_{l}(k) \sim k(l=1,2, \ldots)$ due to the Coulomb-like pion propagator. Consequently, one could also have bound states in partial waves other than ${ }^{3} S_{1}-{ }^{3} D_{1}$ (the deuteron channel). However, we did not fi nd other bound states. Last but not least, we found smaller (in magnitude) and more natural values for the two S-wave scattering lengths in the chiral limit,

$$
a_{\text {c.l. }}\left({ }^{1} S_{0}\right)=-4.1 \pm 1.6_{-0.4}^{+0.0} \mathrm{fm}, \quad a_{\text {c.l. }}\left({ }^{3} S_{1}\right)=1.5 \pm 0.4{ }_{-0.3}^{+0.2} \mathrm{fm} .
$$


The pion mass dependence of the scattering lengths and their inverse is shown in Fig. ฤ. As can be seen from that fi gure, one needs lattice data for pion masses below $300 \mathrm{MeV}$ to perform a stable interpolation to the physical value of $M_{\pi}$. We conclude that nuclear physics in the chiral limit is much more natural than in the real world.
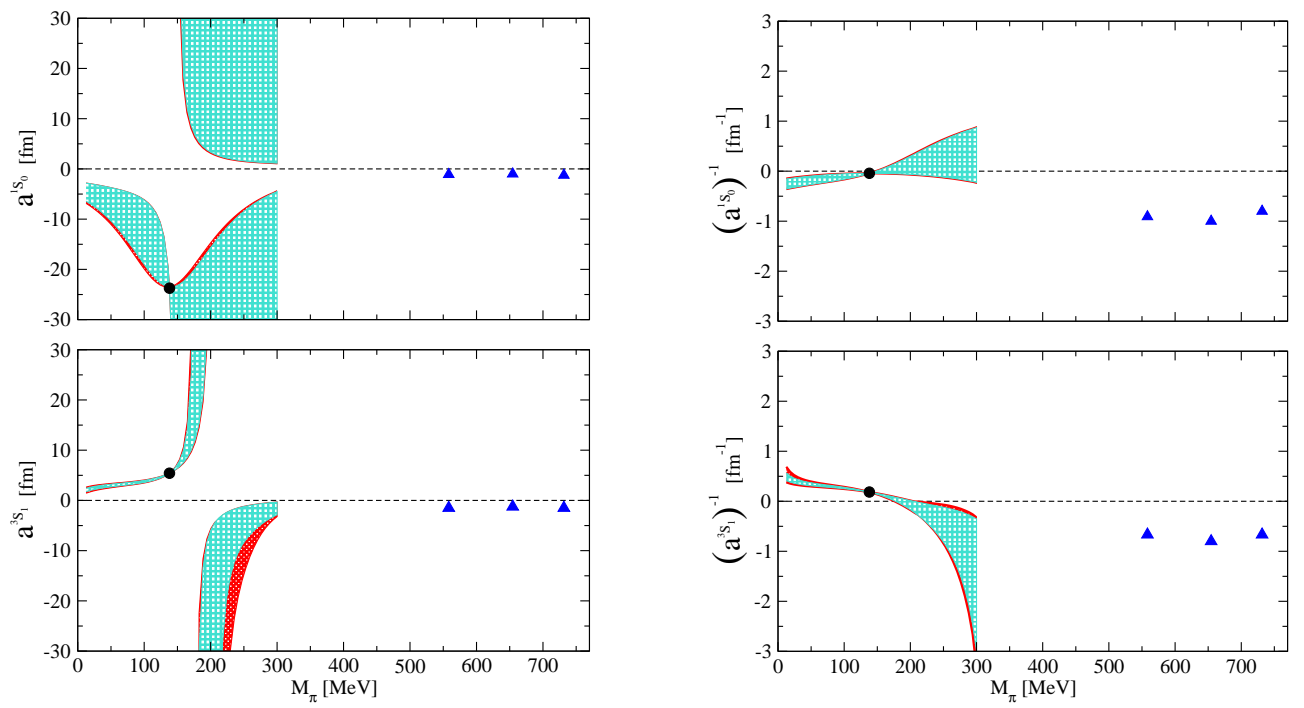

Figure 7: Left panel: The S-wave scattering lengths as functions of pion mass. The shaded areas represent the allowed values according to our analysis. The light shaded band corresponds to our main result with $\bar{d}_{16}=-1.23 \mathrm{GeV}^{-2}$ and the uncertainty due to the unknown LECs $\bar{D}_{S, T}$. The dark shaded band gives the uncertainty if, in addition to variation of $\bar{D}_{S, T}$, the LEC $\bar{d}_{16}$ is varied in the range from $\bar{d}_{16}=-0.91 \mathrm{GeV}^{-2}$ to $\bar{d}_{16}=-1.76 \mathrm{GeV}^{-2}$. The heavy dots corresponds to the values in the real world. The triangles refer to lattice QCD results from [70]. Left panel: The S-wave inverse scattering lengths as functions of the pion mass. Notation as before.

\section{Summary and outlook}

In this talk, I have discussed the foundations and some applications of the quark mass dependence of baryon properties, based on chiral perturbation theory and extensions thereof. This can be summarized as follows:

- Baryon CHPT is a mature fi eld in the light quark sector. For the light quark flavors up and down, the explicit symmetry breaking is generally small at the physical point. This leads to the observation that fourth-order, one-loop calculations provide accurate and unambiguous extrapolation functions for lattice QCD. In the three flavor sector, the situation is less satisfactory, nonetheless a variety of observables can be studied without resorting to resummation techniques, coupled channel analysis or models. The formalism for a covariant inclusion of matter fi elds for spin-1/2 and spin-3/2 fi elds exist. Because of decoupling, only the ground state baryons can contribute to the leading non-analytic terms in the chiral expansion. If one explicitely includes the spin-3/2 decuplet, one must therefore respect decoupling, as discussed in Sec. 1 . 
- It is important to realize that observables are linked by general operator structures. Stated differently, the low-energy constants (LECs) are universal, they contribute to different processes. For that reason and given the present scarcity of low pion mass lattice simulations, one should perform global fi ts to observables combined with input from phenomenology. The quark mass dependence of any observable is given in terms of loop contributions and local operators parameterized by class II LECs, the so-called symmetry breakers. The dynamical (class I) LECs corresponding to operators that do not vanish in the chiral limit can best be determined by phenomenological analysis. I have tried to stress this issue by considering the determination of some of the dimension two pion-nucleon LECs, their appearance in the nucleon mass and in loop corrections to the nucleon isovector magnetic moment. When considering three flavor CHPT, it is important to realize that the SU(3) LECs are related to the SU(2) ones by matching conditions - this allows to get some constraints when analyzing baryon octet observables.

- Given the existing lattice data and our present knowledge of the LECs and the quark mass dependence of important input parameters like the pion decay constant or the axial-vector coupling constant, I conclude that CHPT gives chiral extrapolation functions with a small and moderate theoretical error for $M_{\pi} \lesssim 400$ and $500 \mathrm{MeV}$, respectively. However, in certain cases a smaller window of stability arises at a given order in the chiral expansion, see e.g. the discussion of $g_{A}$ in Sec. 3.3. One of the strengths of the effective fi eld theory approach is the possibility to systematically work out theoretical uncertainties - almost all fi gures shown here underline this important issue.

- Chiral nuclear effective fi eld theory allows to analyze the quark mass dependence of fewnucleon systems, some results for the two-nucleon sector were discussed in Sec. 7 The two-nucleon force appears to be more natural in the chiral limit than for physical quark masses. Also, the observation that both S-wave scattering lengths vanish simultaneously for $M_{\pi} \simeq 175 \mathrm{MeV}$ has led to the speculation of an QCD IR limit cycle in the three-nucleon system [74]. Its relevance for lattice QCD was already stressed by Ken Wilson at Lattice 2004 [75].

- All this can be summarized in one simple sentence: To connect lattice QCD with real QCD (nature) in well-defi ned and precise manner, we need lattice data at low pion masses - most studies performed so far (including the ones presented here) are merely of pioneering nature, eventually paving the way for truly ab initio calculations of baryon properties.

\section{Acknowledgements}

I would like to thank my collaborators, fi rst and most, Véronique Bernard, Thomas Hemmert, Matthias Frink and Evgeny Epelbaum. I would also like to take the opportunity to thank the organizers for setting up such an interesting conference. Useful communications from Claude Bernard, Meinulf Göckeler and Gerrit Schierholz are also acknowledged. 


\section{References}

[1] S. Weinberg, Phenomenological lagrangians, Physica A96 (1979) 327.

[2] J. Gasser and H. Leutwyler, Chiral perturbation theory to one loop, Ann. Phys. 158 (1984) 142.

[3] J. Gasser and H. Leutwyler, Chiral perturbation theory: Expansions in the mass of the strange quark, Nucl. Phys. B250 (1985) 465.

[4] U. G. Meissner, Recent developments in chiral perturbation theory, Rept. Prog. Phys. 56 (1993) 903-996, hep-ph/9302247].

[5] G. Ecker, Chiral perturbation theory, Prog. Part. Nucl. Phys. 35 (1995) 1-80, hep-ph/9501357.

[6] V. Bernard, N. Kaiser, and U.-G. Meissner, Chiral dynamics in nucleons and nuclei, Int. J. Mod. Phys. E4 (1995) 193-346, hep-ph/9501384].

[7] A. Pich, Chiral perturbation theory, Rept. Prog. Phys. 58 (1995) 563-610, hep-ph/9502366.

[8] U.-G. Meissner, Chiral qcd: Baryon dynamics, hep-ph/0007092.

[9] H. Leutwyler, On the foundations of chiral perturbation theory, Ann. Phys. 235 (1994) 165-203, [hep-ph/9311274].

[10] J. Gasser and A. Zepeda, Approaching the chiral limit in qcd, Nucl. Phys. B174 (1980) 445.

[11] N. Fettes, U.-G. Meissner, M. Mojzis, and S. Steininger, The chiral effective pion nucleon lagrangian of order p**4, Ann. Phys. 283 (2000) 273-302, [hep-ph/0001308].

[12] J. Gasser, Light-quark dynamics, hep-ph/0312367.

[13] V. Bernard, T. R. Hemmert, and U.-G. Meissner, Cutoff schemes in chiral perturbation theory and the quark mass expansion of the nucleon mass, Nucl. Phys. A732 (2004) 149-170, hep-ph/0307115.

[14] U.-G. Meissner, Chiral dynamics with strange quarks, AIP Conf. Proc. 717 (2004) 656-664, [hep-ph/0309248].

[15] B. Borasoy, Su(3) chiral effective field theories: A status report, Nucl. Phys. A754 (2005) 191-201, [hep-ph/0402292].

[16] S. Steininger, U.-G. Meissner, and N. Fettes, On wave function renormalization and related aspects in heavy fermion effective field theories, JHEP 09 (1998) 008, [hep-ph/9808280].

[17] T. Becher and H. Leutwyler, Baryon chiral perturbation theory in manifestly lorentz invariant form, Eur. Phys. J. C9 (1999) 643-671, [hep-ph/9901384].

[18] P. Buettiker and U.-G. Meissner, Pion nucleon scattering inside the mandelstam triangle, Nucl. Phys. A668 (2000) 97-112, hep-ph/9908247.

[19] N. Fettes, U.-G. Meissner, and S. Steininger, Pion nucleon scattering in chiral perturbation theory. $i$ : Isospin-symmetric case, Nucl. Phys. A640 (1998) 199-234, [hep-ph/9803266].

[20] V. Bernard, N. Kaiser, and U.-G. Meissner, Determination of the low-energy constants of the next-toleading order chiral pion nucleon lagrangian, Nucl. Phys. A615 (1997) 483-500, [hep-ph/9611253].

[21] M. C. M. Rentmeester, R. G. E. Timmermans, and J. J. de Swart, Determination of the chiral coupling constants $c(3)$ and $c(4)$ in new p p and $n$ p partial-wave analyses, Phys. Rev. C67 (2003) 044001, [nucl-th/0302080]. 
[22] E. Epelbaum, W. Gloeckle, and U.-G. Meissner, Improving the convergence of the chiral expansion for nuclear forces. i: Peripheral phases, Eur. Phys. J. A19 (2004) 125-137, [nucl-th/0304037].

[23] D. R. Entem and R. Machleidt, Accurate charge-dependent nucleon-nucleon potential at fourth order of chiral perturbation theory, Phys. Rev. C68 (2003) 041001, nucl-th/0304018].

[24] N. Fettes and U.-G. Meissner, Pion nucleon scattering in chiral perturbation theory. ii: Fourth order calculation, Nucl. Phys. A676 (2000) 311, hep-ph/0002162].

[25] J. A. McGovern and M. C. Birse, On the absence of fifth-order contributions to the nucleon mass in heavy-baryon chiral perturbation theory, Phys. Lett. B446 (1999) 300-305, hep-ph/9807384.

[26] C. W. Bernard et al., The qcd spectrum with three quark flavors, Phys. Rev. D64 (2001) 054506, [hep-lat/0104002].

[27] CP-PACS Collaboration, A. Ali Khan et al., Light hadron spectroscopy with two flavors of dynamical quarks on the lattice, Phys. Rev. D65 (2002) 054505, hep-lat/0105015.

[28] C. Aubin et al., Light hadrons with improved staggered quarks: Approaching the continuum limit, Phys. Rev. D70 (2004) 094505, [hep-lat/ 0402030 ].

[29] M. Procura, T. R. Hemmert, and W. Weise, Nucleon mass, sigma term and lattice qcd, Phys. Rev. D69 (2004) 034505, [hep-lat/0309020].

[30] QCDSF-UKQCD Collaboration, A. Ali Khan et al., The nucleon mass in $n(f)=2$ lattice qcd: Finite size effects from chiral perturbation theory, Nucl. Phys. B689 (2004) 175-194, [hep-lat/0312030].

[31] S. R. Beane, In search of the chiral regime, Nucl. Phys. B695 (2004) 192-198, [hep-lat/0403030].

[32] V. Bernard, N. Kaiser, and U. G. Meissner, Critical analysis of baryon masses and sigma terms in heavy baryon chiral perturbation theory, Z. Phys. C60 (1993) 111-120, hep-ph/9303311].

[33] M. Frink and U.-G. Meissner, Chiral extrapolations of baryon masses for unquenched three-flavor lattice simulations, JHEP 07 (2004) 028, [hep-lat/0404018].

[34] M. Frink, U.-G. Meissner, and I. Scheller, Baryon masses, chiral extrapolations, and all that, Eur. Phys. J. A24 (2005) 395-409, hep-lat/0501024].

[35] B. Borasoy and U.-G. Meissner, Chiral expansion of baryon masses and sigma-terms, Annals Phys. 254 (1997) 192-232, hep-ph/9607432.

[36] S. Weinberg, The problem of mass, Trans. New York Acad. Sci. 38 (1977) 185-201.

[37] G. Muller and U.-G. Meissner, Virtual photons in baryon chiral perturbation theory, Nucl. Phys. B556 (1999) 265-291, [hep-ph/9903375].

[38] J. Gasser, Hadron masses and sigma commutator in the light of chiral perturbation theory, Ann. Phys. 136 (1981) 62.

[39] B. C. Tiburzi and A. Walker-Loud, Strong isospin breaking in the nucleon and delta masses, hep-lat/0501018.

[40] A. Duncan, E. Eichten, and H. Thacker, Electromagnetic splittings and light quark masses in lattice qcd, Phys. Rev. Lett. 76 (1996) 3894-3897, hep-lat/9602005.

[41] T. R. Hemmert and W. Weise, Chiral magnetism of the nucleon, Eur. Phys. J. A15 (2002) 487-504, [hep-lat/0204005]. 
[42] J. D. Ashley, D. B. Leinweber, A. W. Thomas, and R. D. Young, Nucleon electromagnetic form factors from lattice qcd, Eur. Phys. J. A19 (2004) 9-14, hep-lat/0308024.

[43] QCDSF Collaboration, M. Gockeler et al., Nucleon electromagnetic form factors on the lattice and in chiral effective field theory, Phys. Rev. D71 (2005) 034508, hep-lat/0303019.

[44] T. R. Hemmert, M. Procura, and W. Weise, Quark mass dependence of the nucleon axial-vector coupling constant, Phys. Rev. D68 (2003) 075009, [hep-lat/0303002].

[45] S. R. Beane and M. J. Savage, Baryon axial charge in a finite volume, Phys. Rev. D70 (2004) 074029, [hep-ph/0404131].

[46] A. Fuhrer, The nucleon in finite volume (master thesis, univ. bern), .

[47] D. B. Leinweber, A. W. Thomas, and R. D. Young, Extrapolation of lattice qcd results beyond the power- counting regime, Nucl. Phys. A755 (2005) 59-70, hep-lat/0501028].

[48] D. B. Leinweber, A. W. Thomas, and R. D. Young, Physical nucleon properties from lattice qcd, Phys. Rev. Lett. 92 (2004) 242002, [hep-lat/0302020].

[49] B. Kubis and U.-G. Meissner, Low energy analysis of the nucleon electromagnetic form factors, Nucl. Phys. A679 (2001) 698-734, [hep-ph/0007056].

[50] G. Colangelo and S. Durr, The pion mass in finite volume, Eur. Phys. J. C33 (2004) 543-553, [hep-lat/0311023].

[51] J. Bijnens, G. Colangelo, G. Ecker, J. Gasser, and M. E. Sainio, Pion pion scattering at low energy, Nucl. Phys. B508 (1997) 263-310, hep-ph/9707291].

[52] V. Bernard and U.-G. Meißner, forthcoming, .

[53] J. Kambor and M. Mojzis, Field redefinitions and wave function renormalization to o( $p^{* * 4)}$ in heavy baryon chiral perturbation theory, JHEP 04 (1999) 031, hep-ph/9901235.

[54] N. Fettes, V. Bernard, and U. G. Meissner, One-loop analysis of the reaction pi $n->$ pi pi $n$, Nucl. Phys. A669 (2000) 269-330, hep-ph/9907276].

[55] E. Jenkins and A. V. Manohar, Chiral corrections to the baryon axial currents, Phys. Lett. B259 (1991) 353-358.

[56] T. R. Hemmert, B. R. Holstein, and J. Kambor, Chiral lagrangians and delta(1232) interactions: Formalism, J. Phys. G24 (1998) 1831-1859, hep-ph/9712496.

[57] V. Bernard, T. R. Hemmert, and U.-G. Meissner, Infrared regularization with spin-3/2 fields, Phys. Lett. B565 (2003) 137-145, hep-ph/0303198].

[58] C. Hacker, N. Wies, J. Gegelia, and S. Scherer, Including the delta(1232) resonance in baryon chiral perturbation theory, hep-ph/0505043.

[59] V. Bernard, T. R. Hemmert, and U.-G. Meissner, Chiral extrapolations and the covariant small scale expansion, Phys. Lett. B622 (2005) 141-150, [hep-lat/0503022].

[60] J. M. Zanotti, D. B. Leinweber, W. Melnitchouk, A. G. Williams, and J. B. Zhang, Hadron properties with flic fermions, hep-lat/0407039.

[61] V. Bernard, H. W. Fearing, T. R. Hemmert, and U. G. Meissner, The form factors of the nucleon at small momentum transfer, Nucl. Phys. A635 (1998) 121-145, hep-ph/9801297.

[62] N. Fettes and U. G. Meissner, Pion nucleon scattering in an effective chiral field theory with explicit spin-3/2 fields, Nucl. Phys. A679 (2001) 629-670, [hep-ph/0006299]. 
[63] G. Schierholz, private communication, .

[64] J. Gasser, H. Leutwyler, and M. E. Sainio, Sigma term update, Phys. Lett. B253 (1991) 252-259.

[65] V. E. Lyubovitskij, T. Gutsche, A. Faessler, and E. G. Drukarev, Sigma-term physics in the perturbative chiral quark model, Phys. Rev. D63 (2001) 054026, [hep-ph/ 0009341 ].

[66] I. P. Cavalcante, M. R. Robilotta, J. Sa Borges, D. de O. Santos, and G. R. S. Zarnauskas, Pion delta sigma-term, hep-ph/0507147.

[67] A. Walker-Loud, Octet baryon masses in partially quenched chiral perturbation theory, Nucl. Phys. A747 (2005) 476-507, hep-lat/0405007].

[68] B. C. Tiburzi and A. Walker-Loud, Decuplet baryon masses in partially quenched chiral perturbation theory, Nucl. Phys. A748 (2005) 513-536, [hep-lat/0407030].

[69] E. Epelbaum, U.-G. Meissner, and W. Gloeckle, Nuclear forces in the chiral limit, Nucl. Phys. A714 (2003) 535-574, nucl-th/0207089].

[70] M. Fukugita, Y. Kuramashi, M. Okawa, H. Mino, and A. Ukawa, Hadron scattering lengths in lattice qcd, Phys. Rev. D52 (1995) 3003-3023, hep-lat/9501024].

[71] S. R. Beane and M. J. Savage, Variation of fundamental couplings and nuclear forces, Nucl. Phys. A713 (2003) 148-164, hep-ph/0206113.

[72] E. Epelbaum, U. G. Meissner, W. Gloeckle, and C. Elster, Resonance saturation for four-nucleon operators, Phys. Rev. C65 (2002) 044001, nucl-th/0106007].

[73] S. R. Beane and M. J. Savage, The quark mass dependence of two-nucleon systems, Nucl. Phys. A717 (2003) 91-103, nucl-th/0208021].

[74] E. Braaten and H. W. Hammer, An infrared renormalization group limit cycle in qcd, Phys. Rev. Lett. 91 (2003) 102002, nucl-th/0303038.

[75] K. G. Wilson, The origins of lattice gauge theory, Nucl. Phys. Proc. Suppl. 140 (2005) 3-19, [hep-lat/0412043]. 